\title{
La actitud religiosa y su relación con las distorsiones cognitivas en una muestra de personas pertenecientes a la orientación religiosa de origen protestante
}

The religious attitude and it correlation with the cognitive distortions in a sample of people belonging to the religious orientation of Protestant origin

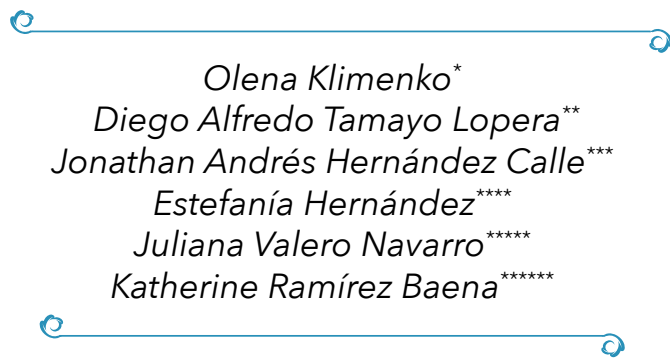

Recibido 02. 03. $2019 \bullet$ Arbitrado 15. 04. $2019 \bullet$

Aprobado 23.05. 2019

* Psicóloga, Magister en Ciencias Sociales, Doctora en Psicopedagogía, Docente Institución Universitaria de Envigado, olenak45@gmail.com, ORCID: 0000-0002-8411-1263

* Psicólogo, Magister en Neuropsicología, Docente tiempo completo, Institución Universitaria de Envigado, datamayol@correo.iue. edu.co, ORCID: 0000-0001-7996-8871

*** Psicólogo, Magister en epidemiología, Docente Institución Universitaria de Envigado, jahernandezc@correo.iue. edu.co, ORCID: 0000-0001-7749-1547

**** Psicóloga, Institución Universitaria de Envigado, estefaniahdz_2611@ hotmail.com

***** Psicóloga, Institución Universitaria de Envigado, valeritto.99@hotmail. com

******Psicóloga, Institución Universitaria de Envigado, krmx@sanvicentefundacion.com

\section{Resumen}

El articulo presenta resultados de estudio orientado a identificar la actitud religiosa y su relación con las distorsiones cognitivas en una muestra de personas que pertenecen a un culto religioso de origen protestante. Investigación de enfoque cuantitativo, nivel descriptivo y corte trasversal, empleo el Inventario de pensamientos automáticos de Ruiz y Lujan (1991) y la escala de Actitud religiosa de Orozco Domínguez (2014) en la muestra intencional de 50 persona pertenecientes a una congregación religiosa de Testigos de Jehová de área metropolitana de Medellín, Colombia. Los resultados indican una alta actitud religiosa en la muestra del estudio, sin presentarse una correlación significativa estadísticamente con las distorsiones cognitivas evaluadas. Sin embargo, aunque no de forma concluyente, los resultados indican la presencia de algunos aspectos importantes como una tendencia hacia mayores puntajes en pensamiento polarizado, sobregeneralización, visión catastrófica, personalización, razonamiento emocional, 
falacia de cambio, culpabilidad y falacia de recompensa divina en el grupo con más alta actitud religiosa, indicando lo anterior, la importancia y la necesidad de continuar con estudios en esta línea con el fin de aproximarse a la comprensión del fenómeno de religiosidad y su relación con aspectos cognitivos del ser humano.

Palabras clave: pensamiento religioso, religión protestante, distorsiones cognitivas, actitudes religiosas.

\section{Abstract}

The article presents results of the study oriented to identify the religious attitude and its relationship with the cognitive distortions in a sample of people that belong to a religious cult of Protestant origin. Investigation with the quantitative approach, descriptive level and cross-section desing use the Inventory of automatic thoughts of Ruiz and Lujan (1991) and the scale of religious Attitude of Orozco Domínguez (2014) in the intentional sample of 50 people belonging to a religious congregation of Witnesses of Jehovah from the metropolitan area of Medellín, Colombia. The results showed a high religious attitude in the study sample without the statistically significant correlation with the cognitive distortions. However, although not conclusively, the results indicated the presence of some important aspects such as a tendency towards higher scores in polarized thinking, overgeneralization, catastrophic vision, personalization, emotional reasoning, fallacy of change, guilt and fallacy of divine reward in the group with highest religious attitude, indicating the importance to continue studies in this line which can help to understand the phenomenon of religiosity and its relationship with cognitive aspects.

Key words: religious thought, protestant religion, cognitive distortions.

\section{Introducción}

El comportamiento religioso representa una de las características propia del ser humano (Valderas, 2013), siendo éste una especie de vida inteligente y analítica que busca explicación a los hechos de la naturaleza y del universo. Históricamente, la religión y la ciencia han sido dos posturas explicativas que mediatizan la comprensión de la realidad para el ser humano (Udías Vallina, 2010).

La religión es un fenómeno social que se desarrolla de forma grupal y está conformada por una masa social, las personas se integran en una religión para constituir elementos básicos en la construcción de su identidad y la concepción básica de la vida y del mundo (cosmogonía) (Pérez-Agote, 2016). 
Actualmente en el campo de la investigación de la psicología social de la religión hay estudios que dan cuenta de los efectos positivos que se presentan a partir de las actividades asociadas a la afiliación religiosa, tales como pertenecer a grupos de oración o congregaciones, siendo estas una fuente de apoyo psicológico e instrumental importante. Adicionalmente se ha sugerido que la actividad religiosa provee un tipo único de apoyo social al relacionar a los individuos con los aspectos inexplicables e incontrolables de la vida diaria. Algunos autores exponen que la religión ofrece un sentido de propósito y, además, la asistencia a una congregación brinda sentimientos de autoestima positiva y control, como resultado de actividades de devoción públicas y privadas, tales como ritos religiosos, oración y meditación al establecer y sostener una relación personal con un ser divino (Pérez, Sandino y Gómez, 2005). Estudios indican, igualmente, que la religion puede actuar como un regulador del comportamiento humano, ayudando, al parecer, a tener un mayor autocontrol, autoregulacion y una mayor persistencia y tenacidad en el logro de los objetivos importantes para personas y sus grupos religiosos (McCullough \& Willoughby, 2009). La religion ha sido tambien asociada con menores indices de depresion (Perez et al., 2005), con un mejor proceso de afrontamiento del duelo (Yoffe, 2015), siendo un factor promotor de salud mental, sobre todo en la poblacion de tercera edad (Valiente-Barroso y García-García, 2010; Miri, Salehiniya, Tiyuri, Bahlgerdi \& Taghizadeh, 2015). Existe una gran cantidad de agrupaciones religiosas benéficas según sus actividades e incidencia en la comunidad y formación de sus miembros, que adelantan actividades de alfabetización, luchas contra SIDA, pobreza extrema y otras problemáticas psicosociales e impulsando cambios sociales en las comunidades (Pérez, 2015; Pérez-Rayón, 2009) y que pueden ser comprendidas como minorías activas (Moscovichi, 1996).

Por otro lado, y en contraste con los aspectos positivos que se encuentran en la religión, existen efectos negativos asociados a la orientación y práctica religiosa. Braam et al. (1998) afirman que la inmersión a ciertas instituciones religiosas puede aumentar el pesimismo e incluso provocar depresión. De igual manera algunas religiones, como el cristianismo, son estrictas y autoritarias, exigen altos niveles de dependencia y sacrificios a cambio de "protección divina", en estas se enfatiza la sensación de desvalimiento y culpa, las creencias religiosas también pueden llegar a sobrepasar el límite de la vida cotidiana normal e incluso colocar al ser humano en un estado en el que se realicen actos ceremoniales exagerados. A esto se le conoce, según Almendros, Gámez, Carrobles y Rodríguez (2011), como grupos de manipulación psicológica, sectas o movimientos religiosos y sus manifestaciones que pueden incluir acontecimientos dramáticos como sacrificios colectivos, violencia excesiva, 
intolerancia, entre otros, que pueden poner en riesgo la salud y bienestar de los miembros de un grupo a los intereses particulares de un líder. Algunos de estos grupos o sectas religiosas generan controversia en el medio social por sus ideas, creencias o prácticas que no solo son insustentadas científicamente, sino perjudiciales, como, por ejemplo, la negación al trasplante de órganos o transfusión de sangre por parte de Testigos de Jehová, que han cobrado, incluso, innumerables vidas, incluyendo niños (Sánchez López, 2011). Existen estudios que consideran el fenómeno de grupos o sectas religiosas como un problema de salud pública, semejante al fenómeno de adicción a drogas (Rodríguez, 2000; Vargas, 2001; Jolyon, 2003).

Para explicar mejor los procesos psicológicos implicados en la religión que son inherentes a todos los seres humanos, es importante abordar las emociones, las cogniciones, las actitudes y las conductas que están implicadas en el comportamiento religioso (Caro y Fediakova, 2000). En esta línea se puede indicar que existen emociones, pensamientos, actitudes y conductas que pueden presentarse de forma disfuncional, patológica o, por el contrario, saludables o constructivos, los procesos cognitivos guían el procesamiento de la información hacía, entre y desde los esquemas con la finalidad de generar los productos o eventos cognitivos. Se ha reconocido que los esquemas nucleares de las personas se relacionan directamente con la identidad, dado que aportan las ideas centrales de sí mismos y de los demás (Lemos, Londoño y Zapata, 2007).

En este asepcto, James \& Wells (2003) resaltan que las relaciones entre las variables religiosas y la salud mental pueden depender de mecanismos cognitivos-conductuales. Es probable que la orientación religiosa de un individuo esté asociada con la formación de modelos mentales religiosos, entendiéndose estos, como un sistema de creencias genéricas que contribuye a las evaluaciones, en particular, relativas a los acontecimientos estresantes de la vida. Asimismo, toda esta serie de atribuciones y contenidos cognitivos que se colocan en torno a la religión, pueden influir positiva o negativamente en el sentido de significado y control sobre los acontecimientos que poseen los individuos, además, la religión puede ser utilizada como defensa psicológica (estrategia de afrontamiento), modelo mental genérico consistente en esquemas que influyen en las evaluaciones y afectan el bienestar modelando la conducta a través de prácticas realizadas para mantener el esquema.

Según Barrett \& Zahl (2013), las creencias y las experiencias religiosas pueden explicarse a partir de estructuras y procesos cognitivos comunes (es decir, no exclusivos de la religión) que son compartidos por todos los humanos. Diferencias individuales en las creencias y expresiones religiosas pueden ser 
explicadas por variaciones en los estilos de procesamiento (por ejemplo, no reflexivo versus reflexivo) y contenido representativo (prevalencia de contenido afectivo en conceptos y esquemas).

En este orden de ideas, es necesario resaltar que el pensamiento humano se puede caracterizar por un enfoque de "dos sistemas" o "procesamiento dual" (Stanovich \& West, 2000; Tremlin, 2006): el primero es el sistema no reflexivo o intuitivo, se caracteriza por ser rápido, automático, aparentemente sin esfuerzo, y predominantemente emocional; y el segundo, es el sistema de razonamiento reflexivo, más lento, deliberado, con esfuerzo, y más racional. Estos dos sistemas no son totalmente independientes, ya que las producciones automáticas del sistema intuitivo sirven como presunciones predeterminadas para el sistema reflexivo. A menos que el sistema reflexivo modifique o anule el sistema intuitivo, este último tiene tendencia a impulsar nuestro pensamiento. Al respecto de lo anterior, Barrett \& Zahl (2013) afirman que la comprensión de la psicología de la religión podría mejorarse al advertir cómo las representaciones religiosas derivadas de nuestro sistema intuitivo, no reflexivo, informan y restringen el pensamiento reflexivo y las conductas consiguientes. Según Boyer (2003), estas representaciones religiosas son adoptadas, en la gran mayoría de los casos, temprano en la vida, integrándose en el razonamiento automático intuitivo y, por lo tanto, sirven como supuestos predeterminados para el posterior aprendizaje y razonamiento. Algunos autores indican que las creencias religiosas pueden programar la mente para formar pensamientos y creencias irracionales aumentando la culpabilidad y distorsiones cognitivas (Demaria \& Kassinove, 1988).

En este aspecto, las distorsiones cognitivas como alteraciones en el procesamiento de información que llevan a las personas a sacar conclusiones arbitrarias al respecto de distintos eventos o situaciones experimentadas, han sido relacionadas con las representaciones y creencias religiosas, empezando por Albert Ellis, quien consideraba que la religión puede tener relación con el pensamiento irracional (1968).

Por otro lado, Amirsardari, Azari \& Esmali Kooraneh (2014) identificaron que una mayor orientación religiosa extrínseca aumenta la distorsión cognitiva, relacionándose con más alto grado de ansiedad y mayores creencias irracionales, sin embargo, la orientación religiosa intrínseca no indica relación con distorsiones cognitivas. AliAkbari, Oraki \& Barghi (2010) indican, igualmente, que las personas con orientación religiosa intrínseca son cognitivamente más racionales tienen mayor salud mental y están más satisfechos con la vida. En la misma línea, algunos autores resaltan que las actitudes religiosas no necesariamente están relacionadas con distorsiones cognitivas, 
ya que las creencias religiosas de una persona pueden tanto causar o exacerbar problemas emocionales, como pueden ser utilizados para mejorar estos problemas, ya que las mismas enseñanzas religiosas pueden ser utilizadas en terapia como guía para reconocer creencias irracionales (Warnock, 1989).

En este orden de ideas es importante tener en cuenta también que distintas orientaciones religiosas están basadas en diferentes representaciones y concepciones doctrinales sobre el ser humano, su respectivo comportamiento, y en general sobre aspectos cruciales de la vida como sexualidad, muerte, valores morales, etc. En este aspecto, existe una gran variedad de orientaciones religiosas (Smith, 2011), que a su vez, luchan por atraer adeptos y extender su influencia, siendo algunas más radicales que otras (Andrade, 2002). En este aspecto, los estudios indican que la orientación religiosa tanto intrínseca como extrínseca, actúa como predictor de fundamentalismo religioso que causa comportamientos extremos de violencia y discriminación (González Villanueva y Reyes Lagunes, 2015).

En este aspecto la orientación religiosa de origen protestante ha sido más asociada a los movimientos fundamentalistas, además del islam (Serrano y Patiño, 2007). En Colombia las dinámicas de expansión de misiones protestantes se han desarrollado bajo una lógica que pretendía consolidar la concepción de una iglesia protestante como contrapropuesta en relación con la iglesia católica, un factor importante es representado por la expansión misionera de estas religiones en el territorio nacional que fue posible debido a las condiciones políticas favorables percibidas por los misioneros en los años 30 (López Amaya, 2014). La globalización y la transnacionalización abrieron las condiciones propicias para fortalecer la influencia de algunas organizaciones internacionales de origen protestante en América Latina, posibilitando su expansión en el país, brindando la alternativa de la salvación espiritual en un vínculo directo con la población y en un sector demográfico en el cual las condiciones de exclusión y pobreza eran predominantes y en donde la iglesia católica atravesaba por una época de desencanto, aumentando, de esta forma, la receptividad la población hacia este tipo de orientaciones religiosas (Duque Daza, 2010). Actualmente en Colombia, donde 94\% de la poblacion se declara creyente, se cuenta con mas de 15000 iglesias y un rapido crecimiento de cantidad de adeptos (Bolivar, 2016).

El presente estudio se orientó a indagar por la actitud religiosa y su relación con las distorsiones cognitivas y la variable de género en una muestra de personas pertenecientes a orientación religiosa de origen protestante, siendo este tema poco estudiado, especialmente en personas asistentes a este tipo de congregaciones. 


\section{Metodología}

Estudio de enfoque cuantitativo observacional trasversal.

\section{Participantes:}

La muestra estuvo compuesta por 50 participantes quienes fueron seleccionados a conveniencia teniendo en cuenta que tuvieran una edad igual o mayor a 18 años, que supieran leer y escribir, que pertenecieran a una congregación religiosa de origen protestante de forma activa en el área metropolitana del Valle de Aburra, y que dieran su consentimiento para participar en el estudio.

\section{Instrumentos}

Para identificar las distorsiones cognitivas se empleó el Inventario de pensamientos automáticos de Ruiz y Lujan (1991), que mide un total de 15 distorsiones cognitivas: abstracción selectiva, pensamiento polarizado, sobregeneralización, interpretación de pensamiento, visión catastrófica, personalización, falacia de control, falacia de justicia, falacia de cambio, razonamiento emocional, etiquetas globales, culpabilidad, deberías, tener razón y falacia de recompensa divina. El Inventario de Pensamientos Automáticos fue aplicado previamente por Ruiz y Lujan (1991) en una muestra mexicana con posterior adaptación a la población peruana por Riofrio \& Villegas (2015), con una confiabilidad según Alfa de Cronbach de 0,945. El instrumento consta de 45 ítems, con escala de opciones de 0 a 3 , donde su respectivo significado es: $0=$ nunca pienso eso; $1=$ algunas veces lo pienso; $2=$ bastantes veces lo pienso; 3 = con mucha frecuencia lo pienso.

Para la medición de la actitud religiosa de los participantes fue implementada "La escala de actitud religiosa" de Orozco-Parra y Domínguez-Espinosa (2014), compuesta por 18 reactivos, con opciones entre totalmente de acuerdo (5) a totalmente en desacuerdo (1), validada en la población mexicana, el Alfa de Cronbach de 0.94 y la varianza explicada en una dimensión del $55.9 \%$, lo que proporciona instrumento con características psicométricas adecuadas.

\section{Procedimiento}

Se realizó un acercamiento a una congregación de orientación religiosa protestante seleccionada a conveniencia, y se invitó a participar del estudio a las personas que cumplieran con los criterios de inclusión de la muestra, y, 
a quienes diligenciaron el consentimiento informado de manera voluntaria se les aplicó el inventario de pensamientos automáticos (Ruiz \& Lujan, 1991) y la escala de actitud religiosa (Orozco-Parra y Domínguez-Espinosa, 2014).

\section{Análisis de datos}

Se verificó la normalidad de los datos mediante la prueba Kolmogorov Smirnov, teniendo las variables del estudio una distribución distinta a la normal ( $\mathrm{p}<0.05)$. Se realizó la recategorización de la variable de actitud religiosa en dos variables cualitativas con menor y mayor actitud, siendo el grupo de menor actitud conformado por sujetos que puntuaron por debajo de una desviación estándar $(\mathrm{M}=69,6 ; \mathrm{DE}=14)$. Se empleó el estadístico de $\mathrm{U}$ de Mann-Whitney para la comparación de distorsiones cognitivas según los dos grupos con mayor y menor actitud religiosa. Los datos se analizaron con el programa estadístico SPSS (Statistical Package for the Social Sciences) en su versión 21.

\section{Resultados}

La muestra general estuvo conformada por cincuenta personas, la edad de los participantes oscilo entre los 19 y 64 años con una edad promedio de 36.03 ( $\mathrm{DE}=12,2$ ), del total de participantes el $64 \%$ fueron mujeres, el 56\% lo constituyen personas con estudios de nivel de bachillerato y un $44 \%$ han cursado estudios universitarios.

Se encontró una media grupal muy alta en la actitud religiosa. Al respecto de las distorsiones cognitivas no se observa la presencia de altos niveles de distorsiones, sin embargo, se encuentran una tendencia hacia un mayor puntaje en las distorsiones cognitivas de falacia de recompensa divina y de visión catastrófica.

Tabla 1: Datos descriptivos de las variables en la muestra del estudio

\begin{tabular}{lcccc}
\hline \multicolumn{1}{c}{ Variable } & Me (Ri) & Min & Max & Puntuación de referencia \\
\hline \multicolumn{1}{c}{ Actitud religiosa } & $75,5(17)$ & 40 & 81 & $17-85$ \\
Filtraje & $1(2)$ & 0 & 9 & $0-9$ \\
Pensamiento polarizado & $1(2)$ & 0 & 4 & $0-9$ \\
Sobre generalización & $1(2)$ & 0 & 6 & $0-9$ \\
Interpretación del pensamiento & $1(1)$ & 0 & 3 & $0-9$ \\
Visión catastrófica & $3(2)$ & 0 & 9 & $0-9$ \\
Personalización & $1(2)$ & 0 & 4 & $0-9$ \\
\hline
\end{tabular}




\begin{tabular}{lcccc}
\hline \multicolumn{1}{c}{ Variable } & Me (Ri) & Min & Max & Puntuación de referencia \\
\hline Falacia de control & $1(2)$ & 0 & 9 & $0-9$ \\
Falacia de justicia & $1(2)$ & 0 & 8 & $0-9$ \\
Razonamiento emocional & $1(3)$ & 0 & 8 & $0-9$ \\
Falacia de cambio & $1(2)$ & 0 & 8 & $0-9$ \\
Etiquetas globales & $1(3)$ & 0 & 8 & $0-9$ \\
Culpabilidad & $1(1)$ & 0 & 8 & $0-9$ \\
Los debería & $1(2)$ & 0 & 8 & $0-9$ \\
Falacia de razón & $1(2)$ & 0 & 3 & $0-9$ \\
Falacia de recompensa divina & $2(2)$ & 0 & 9 & $0-9$ \\
\hline
\end{tabular}

Fuente: Autores

No se encontraron diferencias significativas en las distorsiones cognitivas según grupos de mayor y menor actitud religiosa. Sin embargo, en el grupo de alta actitud religiosa se presentó un mayor puntaje en las siguientes distorsiones cognitivas: pensamiento polarizado, sobregeneralización, visión catastrófica, personalización, razonamiento emocional, falacia de cambio, culpabilidad y falacia de recompensa divina.

Tabla 2: Comparación de variables según los grupos de menor y mayor actitud religiosa

\begin{tabular}{lcccc}
\hline \multicolumn{1}{c}{ Variables } & $\begin{array}{c}\text { Actitud baja } \\
\text { Me (RI) }\end{array}$ & $\begin{array}{c}\text { Actitud alta } \\
\text { Me (RI) }\end{array}$ & $\begin{array}{c}\text { U de Mann } \\
\text { Whitney }\end{array}$ & Valor p \\
\hline Filtraje & $1(3)$ & $1(2)$ & 191 &, 822 \\
Pensamiento polarizado & $0(2)$ & $1(2)$ & 163,5 &, 353 \\
Sobre generalización &, $5(2)$ & $1(2)$ & 173,5 &, 504 \\
Interpretación del pensamiento & $1(1)$ & $1(2)$ & 191 &, 815 \\
Visión catastrófica & $2(2)$ & $3(2)$ & 172 &, 487 \\
Personalización &, $5(2)$ & $1(2)$ & 199 &, 980 \\
Falacia de control & $1(2)$ & $1(2)$ & 175 &, 526 \\
Falacia de justicia & $1(2)$ & $1(2)$ & 198 &, 970 \\
Razonamiento emocional & $1(2)$ & $2(5)$ & 159,5 &, 315 \\
Falacia de cambio & $0(3)$ & $1(2)$ & 169 &, 434 \\
Etiquetas globales & $1(2)$ & $1(4)$ & 167,5 &, 415 \\
Culpabilidad &, $5(3)$ & $1(2)$ & 156 &, 271 \\
Los debería & $1(2)$ & $1(2)$ & 192,5 &, 850 \\
Falacia de razón & $1(2)$ & $1(2)$ & 180 &, 603 \\
Falacia de recompensa divina & $1,5(1)$ & $2(5)$ & 169 &, 438 \\
\hline
\end{tabular}

Fuente: Autores 
La comparación de variables según el género indico una diferencia significativa en la actitud religiosa, siendo la mayor en el género femenino. En cuanto a las distorsiones cognitivas se identificó la diferencia significativa estadísticamente en culpabilidad con mayores puntajes en el género femenino, igualmente. Adicionalmente, aunque no hay diferencia significativa estadísticamente, se puede resaltar una mayor tendencia en el género masculino en cuanto a filtraje y etiquetas globales. En el género femenino, igualmente, sin ser significativa la diferencia, se observó una tendencia hacia mayores puntajes en pensamiento polarizado, visión catastrófica, razonamiento emocional y falacia de recompensa divina.

Tabla 3: Comparación de variables de estudio según los grupos de género

\begin{tabular}{lcccc}
\hline \multicolumn{1}{c}{ Variables } & $\begin{array}{c}\text { Masculino } \\
\text { Me (RI) }\end{array}$ & $\begin{array}{c}\text { Femenino } \\
\text { Me (RI) }\end{array}$ & $\begin{array}{c}\text { U de Mann } \\
\text { Whitney }\end{array}$ & Valor p \\
\hline Actitud religiosa & $58(29)$ & $80(7)$ & 59,5 &, 000 \\
Filtraje & $2(3)$ & $1(2)$ & 221,5 &, 110 \\
Pensamiento polarizado &, $5(3)$ & $1(2)$ & 293,5 &, 893 \\
Sobre generalización & $1(2)$ & $1(2)$ & 293,5 &, 893 \\
Interpretación del pensamiento & $1(2)$ & $1(0)$ & 247,5 &, 264 \\
Visión catastrófica & $2,5(2)$ & $3(3)$ & 290 &, 839 \\
Personalización & $1(2)$ & $1(1)$ & 256,5 &, 363 \\
Falacia de control & $1(2)$ & $1(2)$ & 270 &, 534 \\
Falacia de justicia & $1(2)$ & $1(2)$ & 239 &, 208 \\
Razonamiento emocional & $1(5)$ & $1,5(2)$ & 271,5 &, 563 \\
Falacia de cambio & $1(3)$ & $1(2)$ & 271 &, 550 \\
Etiquetas globales & $1,5(6)$ & $1(3)$ & 246,5 &, 274 \\
Culpabilidad & $1(2)$ & $2(2)$ & 198 &, 037 \\
Los debería & $1(2)$ & $1(2)$ & 256 &, 365 \\
Falacia de razón & $1(2)$ & $0(1)$ & 235 &, 168 \\
Falacia de recompensa divina & $1,5(1)$ & $2(7)$ & 227,5 &, 139 \\
\hline
\end{tabular}

Fuente: Autores

\section{Discusión}

Los datos del estudio indican una actitud religiosa alta en los sujetos de la muestra. Los hallazgos del presente estudio en este aspecto se relacionan con los resultados obtenidos por Avellar, Gouveia, De Souza \& Alves (2013) quienes encontraron en una muestra brasileña una relación entre las creencias protestantes y actitudes religiosas altas, indicando la posibilidad de un mayor 
compromiso de personas afiliados a esta orientación religiosa con los aspectos de comportamiento y pensamiento acorde a sus creencias religiosas.

En cuanto a la diferencia de actitud religiosa entre los géneros, los sujetos de género femenino de la muestra del estudio mostraron una actitud religiosa más alta que el género masculino, presentándose una diferencia significativa estadísticamente. Estos resultados son acorde con hallazgos de otros estudios que indican, igualmente, un mayor nivel de actitud religiosa en mujeres en distintas poblaciones: India (Dutt, 1965); Shrilanka (Kajavinthan, 2015); Puerto Rico (Raffo, 1978); México (Vitelio y Elisondo, 1972; Orozco \& Domínguez, 2014); Inglaterra (Poppleton \& Pilkington, 1963).

Por otro lado, algunos estudios plantean que, aunque las mujeres pueden tener una mayor referencia por religión, no se presenta diferencia significativa estadísticamente en la actitud religiosa entre hombres y mujeres (TinocoAmador, 2009; Baring et al., 2018; Tahmasbipour \& Taheri, 2011).

En este aspecto, es necesario resaltar que un mayor nivel de religiosidad observado durante décadas en las investigaciones, llevo a buscar explicaciones de este fenómeno, entre las cuales está la hipótesis de factores biológicos, como, por ejemplo, relación de niveles más bajos de testosterona con mayor favorabilidad hacia religión (Stark, 2002), o factores de personalidad, donde rasgos de personalidad típicamente femeninas se asocian con más alta religiosidad (Thompson, 1991). Por otro lado, muchos autores respaldan la hipótesis de los factores sociales como una menor participación social y tendencias educativas tradicionalistas orientadas a trasmitir a las mujeres exigencias de mayor religiosidad con el propósito de controlar la sexualidad femenina ( $\mathrm{Tr}$ zebiatowska \& Steve, 2012); igualmente, se consideran los factores psicosociales como mayor inseguridad existencial en la vida que presentan mujeres, relacionada con menores ingresos económicos, vulnerabilidad física, envejecimiento, discriminación, etc., que contribuyen a una mayor religiosidad, siendo esta fuente de apoyo psicológico (Norris \& Inglehart, 2008).

A partir de lo anterior, los autores indican que a medida que estas diferencias sociales entre los géneros se disminuyen, también se equipara la diferencia en la actitud religiosa (Voas, McAndrew \& Storm, 2013). Por ejemplo, hay estudios que indican que las mujeres que trabajan en la fuerza laboral son tres veces menos religiosas que las que estas por fuera del trabajo formal, disminuyendo su diferencia con los hombres (Schnabel, 2016; 2017; 2018). Igualmente, estudios revelan que la brecha en la religiosidad y actitud religiosa es practicante inexistente en muchos países de mayoría musulmana, así como en otros países no cristianos como India y China (Pew Research Center, 2016). 
Los estudios contemporáneos de la religión parecen estar convergiendo cada vez más en un consenso de que la brecha religiosa de género probablemente surge de una complicada combinación de múltiples factores (Sullins, 2006), lo cual puede explicar diferencias de resultados obtenidos en distintos estudios al respecto de diferencias de género en las actitudes religiosas, ya que son realizadas en poblaciones con distintas características sociodemográficas.

$\mathrm{Al}$ respecto de las distorsiones cognitivas no se observó la presencia de altos niveles de distorsiones en el total de las personas participantes, sin embargo, se encuentra una tendencia hacia puntajes más altos en falacia de recompensa divina que se define como la tendencia a no buscar solución a problemas o dificultades suponiendo que la situación mejorará en el futuro mágicamente; y visión catastrófica denominada como el hecho de adelantar acontecimientos de modo catastrófico para los intereses de cada uno (Lemos et al., 2007).

Lo anterior sugiere una posible tendencia de sujetos de la muestra con orientación religiosa protestante hacia una mayor dificultad en la búsqueda de soluciones para la problemática que pueda estar pasando y creencias trágicas en la forma que asimila los sucesos de su vida cotidiana de acuerdo a las distorsiones de falacia de recompensa divina y visión catastrófica, sin que esto implique necesariamente correcta funcionalidad o disfuncionalidad en su relación consigo mismo o con los demás.

En cuanto a la diferencia en las distorsiones cognitivas según la variable sexo, solo se encontró la diferencia significativa estadísticamente en culpabilidad con mayores puntajes en el género femenino. En este aspecto, hay estudios que también indican la presencia de diferencias significativas entre los sexos, con mayores distorsiones cognitivas en mujeres en abstracción, el tremendismo y el razonamiento emocional y en los "debería" en los hombres (Rodríguez-Testal, Carrasco, del Barrio y Catalán, 2002). Otros estudios indican que no identifican diferencias significativas en distorsiones cognitivas según el género (Aguirre (2015), o presentan datos contrarios a los encontrados en el presente estudio, afirmando la presencia de una mayor tendencia en los hombres en cuanto a culpabilidad, falacia de control y razonamiento emocional (Canario y Báez, 2007).

En relación a lo anterior, es necesario considerar las características de la población estudiada en cada uno de los estudios que indagan por diferencia en distorsiones cognitivas según el género, ya que las variables asociadas a las distorsiones cognitivas consideradas en estos estudios, como, por ejemplo, el VIH, depresión, conductas agresivas, etc., al igual como las características de instrumentos empelados, pueden incidir en resultados. 
En cuanto a la relación entre la actitud religiosa y las distorsiones cognitivas, los resultados del estudio indican que no hay una diferencia significativa estadísticamente en las distorsiones cognitivas según los grupos de mayor y menor actitud religiosa.

En este aspecto es importante mencionar que hay estudios que relacionan distorsiones cognitivas con orientación religiosa (AliAkbari Dehkordi, Oraki \& Barghi Irani, 2010; Amirsardari, Azari, \& Esmali, 2014; Díaz \& Negredo, 2016), indicando que la orientación religiosa extrínseca es la que demuestra una mayor relación con estas. Es necesario precisar que la religiosidad intrínseca implica la internalización de las enseñanzas de las religiones y el descubrimiento de motivos maestros personales en la religión, mientras que la religiosidad extrínseca refleja aspectos más instrumentales y utilitarios de la religión, brindando seguridad y consuelo, sociabilidad y distracción, estatus y auto justificación (Hunt \& King, 1971; Allport \& Ross, 1967).

En este aspecto la religiosidad intrínseca estaría relacionada con una implicación y reflexión personal sobre la existencia, cumpliendo el papel orientador de esta, tal como plantean algunos autores, indicando que los conceptos religiosos activan y refuerzan sistemas mentales relacionados con comprensión del mundo e interpretación de los hechos, cumpliendo función normativa, moral y existencial, dándole al practicante un sentido sobre el cual orienta su vivir (Valderas, 2013; Díaz, 2015).

Sin embargo, lo anterior no implica que las creencias religiosas y el grado de la actitud religiosa estarían relacionados con distorsiones cognitivas.

En este aspecto es necesario puntualizar que el estudio de distorsiones cognitivas desde su inicio fue orientado a las poblaciones con problemáticas de carácter clínico como depresión, diferentes trastornos de personalidad, entre otros, orientados más a la compresión de fenómenos psicológicos en el campo de psicopatología (Beck, Rush, Shaw, Emery, 1992). En este sentido, las distorsiones cognitivas reflejan los hábitos de pensar de una persona, la forma como ésta interpreta los hechos y acontecimientos y pueden llevar a desarrollar problemas psicológicos como depresión, fobias, obsesión, entre otros (Dattilio \& Padesky, 2004).

En cuanto a la relación de estas distorsiones con la actitud frente a la religión, los resultados del presente estudio indican que no hay una relación significativa estadísticamente entre la actitud religiosa y distorsiones cognitivas, mostrando que una actitud religiosa alta no necesariamente este asociada con la presencia de distorsiones cognitivas, tal como indica también Warnock (1989). 
En este aspectos se podría suponer que las distorsiones cognitivas no determinan de forma significativa el grado de religiosidad de una persona o sus preferencias religiosas, siendo la religiosidad un fenómeno multicausal y complejo, relacionado con múltiples factores tanto a nivel personal, como psicosocial y cultural (Iranzo Dosdad, 2013).

Aunque los resultados del presente estudio no son del todo concluyentes debido a la limitación en el tamaño de la muestra del estudio, los datos obtenidos sugieren la importancia de continuar en la misma línea de estudios con el fin de contribuir a una mejor comprensión del fenómeno de religiosidad y su relación con los procesos cognitivos. Es importante profundizar en esta línea de estudios con el fin de aproximarse a una posible clasificación de perfiles cognitivos que permitan ampliar la comprensión sobre el fenómeno religioso y su relación con la psicología, ya que, como indican estudios en este tema, orientación religiosa y religiosidad es un factor importante a la hora de comprender los elementos relacionados con los aspectos cognitivos del ser humano (AliAkbari Dehkordi, Oraki \& Barghi Irani, 2010; Amirsardari, Azari, \& Esmali, 2014).

\section{Referencias}

Aguirre, E. (2015). Distorsiones cognitivas y su influencia en las relaciones afectivas de pareja en adolescentes. Trabajo de grado, Psicología, Pontificia Universidad Católica del Ecuador. Disponible en http://repositorio.pucesa.edu.ec/ bitstream/123456789/1173/1/75706.pdf

AliAkbari Dehkordi, M., Oraki, M, Barghi Irani, Z. (2010). Relation between religious orientation with anxiety about death, and alienation in aged peoples (seniors) in Tehran. Social Psychology Research, 1(2): 140-159, https://www.sid.ir/en/ journal/ViewPaper.aspx?id $=263373$

Allport, G., Ross, M. (1967). Personal religious orientation and prejudice. Journal of Personality and Social Psychology, 5(4):432-443, https://pdfs.semanticscholar. org/bfa1/e5d350b1307ca9796f86495af668e9758e4f.pdf

Almendros, C., Gámez Guadix, M., Carrobles, J. A., \& Rodríguez Carballeira, Á. (2011). Abuso Psicológico en Grupos Manipuladores. Psicología Conductual, 19(1), 157-182. Obtenido de https://search.proquest.com/openview/246384b4 Ofd5a26c630ab477ee0c3823/1?pq-origsite $=$ gscholar

Amirsardari, L., Azari, S., Esmali Kooraneh, A. (2014). The relationship between religious orientation, and gender with a cognitive distortion. Iran J Psychiatry 
Behav Sci, 8(3), 84-90. Retrieved from https://www.ncbi.nlm.nih.gov/pmc/ articles/PMC4359730/

Avellar de Aquino, T. A., Gouveia, V. V., de Souza Silva, S., \& Alves de Aguiar, A. (2013). Escala de Atitudes Religiosas, Versão Expandida (EAR-20): Evidências de Validade. Avaliação Psicológica, 12(2), 109-119. Obtenido de http://search. ebscohost.com/login.aspx?direct $=$ true $\& \mathrm{db}=\mathrm{asn} \& \mathrm{AN}=116231527 \&$ lang $=$ es\&site =ehost-live

Baring, R., Sarmiento, F., Sibug, N., Lumanlan, P., Bonus, B., Samia, C., Reysen, S. (2018). Filipino College Students' Attitudes towards Religion: An Analysis of the Underlying Factors. Religions, 9(3): 85-98; https://doi.org/10.3390/rel9030085

Barrett, J., Zahl, B. (2013). Cognition, Evolution, and Religion. In K. I. Pargament (Editor in Chief), APA Handbook of Psychology, Religion, and Spirituality. Washington DC.: American Psychological Association

Beck, A., Rush, J., Shaw, B., Emery, G. (1992). Terapia Cognitiva de la Depresión. Madrid: Editorial Desclée de Brouwer

Boyer, P. (2003). Religious thought and behavior as by-products of brain function. Trends in Cognitive Sciences, 7(3), 119-124, DOI: 10.1016/S1364-6613(03)00031-7

Bolivar, A. (2016). Los rostros otros de las iglesias protestantes en Bogotá. Disponible en https://www.desdeabajo.info/component/k2/item/29263-los-rostros-otrosde-las-iglesias-protestante-en-bogota.html

Braam, A., Beekman, A., Knipscheer, C., Deeg, D., Van Den Eeden, P., Van Tilburg, W. (1998). Religious Denomination and Depression in Older Dutch Citizens: Patterns and Models. Journal of Aging and Health, 10(4), 483-503. https://doi. org/10.1177/089826439801000405

Canario, J., Báez, M. (2007). Distorsiones cognitivas en personas que viven con VIH. Perspectivas psicológicas, 5(5): 49-60, http://pepsic.bvsalud.org/pdf/pp/v5/ v5a07.pdf

Caro, I., Fediakova, E. (2000). Los Fundamentalismos Religiosos: Etapasy Contextos de Surgimiento. Fermentum. Revista Venezolana de Sociología y Antropología, 10(29), 453-467. Obtenido de http://www.redalyc.org/articulo.oa?id=70511228008

Dattilio, F., Padesky, Ch. (2004). Terapia Cognitiva con parejas. Bilbao: Editorial Desclée De Brouwer.

Demaria, T., Kassinove, H. (1988). Predicting guilt from irrational beliefs, religious affiliation and religiosity. Rational-Emot Cognitive-Behav Therapy, 6(4): 259-272, https://doi.org/10.1007/BF01061292 
Díaz, J. (2015). Revelación y moral. Ensayos de filosofía II. Bogotá: Universidad Santo Tomás.

Duque Daza, J. (2010). Las comunidades religiosas protestantes y su tránsito hacia lo político-electoral en Colombia, 1990-2007. Revista Mexicana de Sociología, 72(1), 73-111. https://www.jstor.org/stable/25677032

Dutt, N. (1965). Attitudes of the university students toward religion. Journal of Psychological Researches, 9 (3): 127-130, https://psycnet.apa.org/ record/1966-06569-001

Ellis, A. (1968). Is Objectivism a Religion? Nueva York: L. Stuart

González Villanueva, M., Reyes Lagunes, I. (2015). Orientación Religiosa, Identidad Grupal y Religiosidad como Predictores del Fundamentalismo Religioso. Acta de Investigación Psicológica, 5 (2): 1984-1995, https://doi.org/10.1016/S20074719(15)30018-1

Hunt, R., King, M. (1971). The intrinsic-extrinsic concept: a review and evaluation. Journal for the Scitific Study of Religion, 10(4):339-356, DOI: 10.2307/1384780

Iranzo Dosdad, A. (2013). La comprensión del fenómeno religioso y sus desafíos a las Ciencias Sociales. Revista de Estudios Sociales, 47: 183-190, DOI: http://dx.doi. org/10.7440/res47.2013.15

James, A., Wells, A. (2003). Religion and mental health: Towards a cognitivebehavioural framework. British Journal of Health Psychology, 8: 359-376. doi:10.1348/135910703322370905

Jolyon, W. (2003). Sectas contemporáneas: Imagen utópica, realidad infernal. Cap. 3. En Erdely, J. (editor), Sectas destructivas: Un análisis científico. México: Publicaciones para el Estudio Científico de la Religión.

Kajavinthan, K. (2015). A Study of Religious Attitude among School Students in Jaffna District, Srilanka. Journal Of Humanities And Social Science, 20 (7): 73-76, DOI: 10.9790/0837-20747376

Lemos Hoyos, M., Londoño Arredondo, N. H., Zapata Echavarría, J. A. (2007). Distorsiones cognitivas en personas con dependencia emocional. Informes Psicológicos, 9: 55-69. Obtenido de https://revistas.upb.edu.co/index.php/ informespsicologicos/article/view/1707/1650

López Amaya, J. D. (2014). Misiones protestantes en Colombia 1930-1946. Geografía y política de la expansión evangélico-pentecostal. Anuario Colombiano de Historia Social y de la Cultura, 41(2), 65-103. doi:10.15446/achsc.v41n2.48782 
McCullough, M., Willoughby, B. (2009). Religion, Self-Regulation, and Self-Control: Associations, Explanations, and Implications. Psychological Bulletin, 135 (1): 69 -93, DOI: 10.1037/a0014213

Moscovici, S. (1996). Psicología de las minorías activas. $2^{\text {a }}$ edición. Madrid: Ediciones Morata.

Miri, M., Salehiniya, H., Tiyuri, A., Bahlgerdi, M., Taghizadeh, A. (2015). Relationship between religious orientation and mental health in older people. History of medicine Journal, 6(21): 83-102, http://journals.sbmu.ac.ir/en-mh/article/view/14765

Norris, P., Inglehart, R. (2008). Existential Security and the Gender Gap in Religious Values. Draft chapter for Social Science Research Council conference on Religion \& International Affairs, New York, Feb. 15-16, 2008, Disponible en https:// sites.hks.harvard.edu/fs/pnorris/Acrobat/SSRC\%20The\%20gender\%20gap\%20 in\%20religiosity\%20Norris\%20and\%20Inglehart\%20\%233.pdf

Orozco-Parra, C. L., Domínguez-Espinosa, A. (2014). Diseño y validación de la Escala de Actitud Religiosa. Revista de Psicología Universidad de Chile, 23(1), 3-11. doi:10.5354/0719-0581.2014.32868

Pérez, R. (2015). Actores y discursos religiosos en la protesta social. Conexión, 4 (4): 104-119, http://revistas.pucp.edu.pe/index.php/conexion/article/view/14977

Pérez-Agote, A. (2016). La religión como identidad colectiva: las relaciones sociológicas entre religión e identidad. Papeles del CEIC, vol. 2016/2, papel 155, CEIC (Centro de Estudios sobre la Identidad Colectiva), Universidad del País Vasco, http://dx.doi.org/10.1387/pceic.16178

Pérez, A., Sandino, C., Gómez O., V. (2005). Relación entre Depresión y Práctica Religiosa: Un estudio Exploratorio. Suma Psicológica, 12 (1): 77-86. Obtenido de http://www.redalyc.org/articulo.oa?id=134219072005

Pew Research Center (2016). The Gender Gap in Religion Around the World. Disponible en https://www.pewresearch.org/wp-content/uploads/sites/7/2016/03/ Religion-and-Gender-Full-Report.pdf

Poppleton, P., Pilkington, G. (1963). The Measurement of Religious Attitudes in a University Population. British Journal of Social and Clinical Psychology, 15 (2): 3646, https://doi.org/10.1111/j.2044-8260.1963.tb00372.x

Raffo, J. (1978). La actitud religiosa del estudiante universitario de Puerto Rico. Revista Latinoamericana de Psicología, 10 (3): 387 - 401, Disponible en: http:// www.redalyc.org/articulo.oa?id $=80510307$

Rodríguez, P. (2000). Adicción a Sectas: pautas para el análisis, prevención y tratamiento. Barcelona: Ediciones B. 
Rodríguez-Testal, J., Carrasco, M., del Barrio, M., Catalán, M. (2002). Errores cognitivos en jóvenes y su relación con la sintomatología depresiva. Anuario de Psicología, 33 (3): 409-432, https://www.raco.cat/index.php/AnuarioPsicologia/ article/.../88496

Ruiz, J., Luján, J. (1991). Manual de Psicoterapia Cognitiva. Inventario de pensamientos automáticos. Psicología Online. http://www.psicologia-online.com/ESMUbeda/ Libros/SentirseMejor/sentirse2.htm

Sánchez, López, S. (2011). Tres miradas psicosociales al fenómeno de las sectas religiosas. Revista Científica de Psicología, Ciencias Sociales, Humanidades y ciencias de la Salud, 2 (1): 79-110, https://www.academia.edu/10964592/Tres_ miradas_psicosociales_al_fen\%C3\%B3meno_de_las_sectas_religiosas

Serrano, E., Patino, C. (2007). La fe armada. Bogotá: Intermedio editores.

Schnabel, L. (2016). The Gender Pray Gap: Wage Labor and the Religiosity of High-Earning Women and Men. Gender \& Society. 30(4): 643-669. https://doi. org/10.1177/0891243216644884

Schnabel, L. (2017). Gendered Religiosity. Review of Religious Research. 59(4): 547556, https://doi.org/10.1007/s13644-017-0302-

Schnabel, L. (2018). More Religious, Less Dogmatic: Toward a General Framework for Gender Differences in Religion. Social Science Research. 75(1):58-72, https:// doi.org/10.1016/j.ssresearch.2018.06.010

Smith, H. (2011). Las religiones del mundo. Barcelona: Editorial Kairos.

Stanovich, K. E., West, R. F. (2000). Individual differences in reasoning: Implications for the rationality debate. Behavioral and Brain Sciences, 23, 645-726, Disponible en http://psy2.ucsd.edu/ mckenzie/StanovichBBS.pdf

Stark, R. (2002). Physiology and Faith: Addressing the 'Universal' Gender Difference in Religious Commitment. Journal for the Scientific Study of Religion, 41(3): 495507, https://doi.org/10.1111/1468-5906.00133

Sullins, P. (2006). Gender and Religion: Deconstructing Universality, Constructing Complexity. American Journal of Sociology 112(3):838-880, DOI: 10.1086/507852

Tahmasbipour, N., Taheri, A. (2011). The Investigation of Relationship between Religious Attitude (Intrinsic and Extrinsic) with depression in the university student. Procedia - Social and Behavioral Sciences 30: 712 - 716, doi:10.1016/j. sbspro.2011.10.139 
Tinoco-Amador, J. (2009). Identificando los constructos de la religiosidad para jóvenes universitarios en México. Universitas Psychologica, 8 (3): 807-829, http:// www.scielo.org.co/pdf/rups/v8n3/v8n3a16.pdf

Thompson, E. (1991). Beneath the Status Characteristics: Gender Variations in Religiousness. Journal for the Scientific Study of Religion, 30(4): 381-394, DOI: $10.2307 / 1387275$

Tremlin, T. (2006). Minds and Gods: The cognitive foundations of religion. New York: Oxford University Press.

Trzebiatowska, M., Steve, B. (2012). Why Are Women More Religious Than Men? Published to Oxford Scholarship Online. DOI:10.1093/acprof:oso/9780199608102.001.0001

Udías Vallina, A. (2010). Ciencia y religión. Dos visiones del mundo. Madrid: Ed. Salterrae

Valderas, J. (2013). Neurociencia cognitiva de la religión I. Estudios Filosóficos LXII, 63-97. Obtenido de http://search.ebscohost.com/login.aspx?direct $=$ true $\& \mathrm{db}=\mathrm{fu}$ a\&AN $=87626554 \&$ lang $=$ es\&site $=$ ehost-live

Valiente-Barroso, C., García-García, E. (2010). La religiosidad como factor promotor de salud y bienestar para un modelo multidisciplinar de atención psicogeriátrica. Psicogeriatría, 2 (3): 153-165, https://www.ucm.es/data/cont/docs/140-201310-04-documento25550.pdf

Vargas, M. (2001). Los nuevos grupos religiosos y sectas en el actual sistema social español. Anales de Historia Contemporánea, 17: 501-521, https://dialnet.unirioja. es/descarga/articulo/237443.pdf

Vitelio G., Elisondo F. (1972). Dos encuestas sobre actitudes: modelo de investigación social. México: Trillas.

Voas, D., McAndrew, S., Storm, I. (2013). Modernization and the Gender Gap in Religiosity: Evidence from Cross-National European Surveys. Kölner Zeitschrift für Soziologie und Sozialpsychologie. 65(1): 259-283, https://doi.org/10.1007/ s11577-013-0226-5

Warnock, S. (1989). Rational-emotive therapy and the christian client. Journal of Rational-Emotive and Cognitive-Behavior Therapy, 7 (4): 263-274, DOI: 10.1007 / BF01073812

Yoffe, L. (2015). Afrontamiento religioso espiritual de la pérdida de un ser querido. Avances En Psicología, 23(2): 155-176. Recuperado a partir de http://revistas. unife.edu.pe/index.php/avancesenpsicologia/article/view/160 